\title{
What can X-rays tell us about accretion, mass loss and magnetic fields in young stars?
}

\author{
Thierry Montmerle
}

Laboratoire d'Astrophysique de Grenoble, BP 53X, 38400 St Martin d'Hères, France email: montmerle@obs.ujf-grenoble.fr

\begin{abstract}
Until recently, X-rays from low-mass young stars $\left(10^{5}-10^{6} \mathrm{yr}\right)$ were thought to be a universal proxy for magnetic activity, enhanced by 3-4 orders of magnitude with respect to the Sun, but otherwise similar in nature to all low-mass, late-type convective stars (including the Sun itself). However, there is now evidence that other X-ray emission mechanisms are at work in young stars. The most frequently invoked mechanism is accretion shocks along magnetic field lines ("magnetic accretion"). In the case of the more massive A- and B-type stars, and their progenitors the Herbig AeBe stars, other, possibly more exotic mechanisms can operate: stardisk magnetic reconnection, magnetically channeled shocked winds, etc. In any case, magnetic fields, both on small scale (surface activity) and on large scale (dipolar magnetospheres), play a distinctive role in the emission of X-rays by young stars, probably throughout the IMF.
\end{abstract}

Keywords. accretion disks, line: identification, plasmas, stars: activity, stars: coronae, stars: early-type, stars: magnetic fields, stars: pre-main-sequence, stars: winds, outflows, X-rays: stars

\section{Introduction: early stellar evolution and magnetic fields}

Low-mass stars, and in particular solar-like stars, form as a result of the collapse of an extended protostellar envelope, via the formation of an embedded accretion disk. Such disks live for a few million years, throughout the so-called "classical T Tauri" (CTTS) phase (e.g., Hillenbrand 2006). In the early phases, mass loss is observed to take place in the form of bipolar jets and outflows: this is sometimes called the "accretion-ejection" phenomenon. Although there are significant differences in the proposed theoretical models, it is widely accepted that accretion and ejection are closely coupled via magnetic fields, at least out to spatial scales of a few stellar radii, and perhaps even (in some models) throughout the accretion disk ("disk winds") (e.g., Pudritz et al. 2007).

In contrast, magnetic fields are not thought to play a major role in the early evolution of massive stars, but there is now evidence for their presence in a significant fraction of $\mathrm{O}$ and $\mathrm{B}$ stars, and for their influence on radiative winds. The situation of intermediatemass stars (the so-called "Herbig AeBe" stars, with $M_{\star} \sim 2-3 M_{\odot}$ ), is less clear, but in a few cases there is indirect evidence for large-scale magnetic fields.

Direct measurements and modeling of magnetic fields have made spectacular advances in recent years, thanks mainly to observations of the Zeeman effect via spectropolarimetic measurements and Doppler imaging techniques (e.g., Donati et al. 2007; Strassmeier \& Rice 2006; Yang et al. 2007). However, indirect access to magnetic fields has been provided for a long time by X-ray observations (spectra, timing), based on the idea that the only way to confine a hot plasma $\left(T \sim 10^{6}-10^{7} \mathrm{~K}\right.$, i.e., thermal X-ray energies $\sim 0.1$ to a few keV) is to trap it in closed magnetic loops (e.g., Feigelson \& Montmerle 1999; Güdel 2004; Micela \& Favata 2005). The numbers obtained by the various methods for the surface magnetic field intensities are quite similar: $B_{\star} \approx 0.1-1 \mathrm{kG}$, i.e., comparable to values obtained in present-day solar active regions. 


\section{X-ray emission from young stars: processes and environments}

Stellar X-rays are thermal in the $\sim \mathrm{keV}$ range, and can be produced as the end result of internal structure processes. This is the case for low-mass stars, which have outer convective envelopes. Magnetic fields are currently thought to be generated via the dynamo effect at the bottom of the convective zone (the so-called "tachocline", e.g., Brun \& Zahn 2006), and to buoy out to the surface across the convective zone. Reconnections between magnetic loops of opposite polarity, anchored in the photosphere, result in flaring and sudden heating of the photospheric gas to X-ray temperatures. The prototype of this behavior is the Sun itself, as observed in particular by the Yohkoh satellite (Peres et al. 2004). The X-ray signatures of this stellar "magnetic activity" are: temporal variability over a time scale of a few hours (flares: fast rise followed by slow decay corresponding to cooling); frequent 2-temperature spectra, with a dominant hard component $(T \sim$ a few 10 $\mathrm{MK})$, and a less important soft component $(T \sim$ a few $\mathrm{MK})$; "coronal" plasma densities $\left(n_{e} \sim 10^{10}-10^{11} \mathrm{~cm}^{-3}\right.$ ) (e.g., Wolk et al. 2005). The level of X-ray luminosity (expressed in $L_{X} / L_{b o l}$ ) is 3-4 orders of magnitude higher than in the Sun, in fast rotating stars (like RS CVn binaries) or in fully convective stars (like dMe stars and T Tauri stars).

Stellar X-rays can also be produced by shocks at the photospheric level. This is the case of the winds of massive stars, which have been known for a long time to be radiatively unstable. As a result, myriads of shock waves, with velocities $\sim$ several 100 $\mathrm{km} . \mathrm{s}^{-1}$, criss-cross the wind and emit X-rays (e.g., Kudritzki \& Puls 2000). The X-ray signatures of radiative winds are: no (or small but random) temporal variability over time scales of hours; the overall spectrum is dominated by the weakly absorbed outer layers: consequently, the spectrum is basically soft (sub-keV, or equivalent $T \sim$ a few $\mathrm{MK}$ ), and the plasma densities are comparatively low $\left(n_{e} \sim 10^{9}-10^{11} \mathrm{~cm}^{-3}\right)$ (see, e.g., Owocki \& Cohen 1999). As explained below (§4), when a magnetic field exists and is sufficiently strong to confine the wind within a large-scale closed magnetosphere, the signature is modified, the most important change being the possibility of rotational modulation of the $\mathrm{X}$-ray emission, at the rotational period of the star.

Additional X-ray emission mechanisms are possible, as a result of the interactions between the star and the surrounding medium. The now classical general picture of young low-mass stars is that of a central star postulated to be surrounded by a largescale magnetospheric structure, linking the star and the disk at the corotation radius $R_{c}$ (typically $R_{c} \sim 2-3 R_{\star} \approx 0.05 \mathrm{AU}$ ): beyond $R_{c}$, the disk is in Keplerian rotation. This opens two new possibilities for X-ray emission: (i) magnetic interactions between the star and its circumstellar disk, which will be the topic of $\S 5$; (ii) shock interactions between the jet and the surrounding medium (protostellar envelope close to the star, and/or ambient ISM farther away) (see Güdel, this volume). Fig. 1 summarizes the various X-ray emission regions associated with low- and intermediate-mass stars.

\section{Low-mass stars: magnetic activity}

The X-ray emission from TTS (both "classical", still surrounded by disks, and "weakline", without disks), is extremely well documented, after over 25 years of X-ray observations of star-forming regions. On the one hand, to date many star-forming regions have been observed by various X-ray satellites, with typical "short" exposures of 30-150 ksec, yielding hundreds of individual $\mathrm{T}$ Tauri detections down to the brown dwarf regime. On the other hand, the unique, very long Chandra exposure $(850 \mathrm{ksec})$ of the Orion Nebula Cluster (the so-called "Chandra Orion Ultradeep Project", or COUP; PI E. Feigelson), has yielded in a single observation over 1500 detections of TTS (to which are added detections of massive stars and protostars, see below) over a $17^{\prime} \times 17^{\prime}$ FOV (Getman 


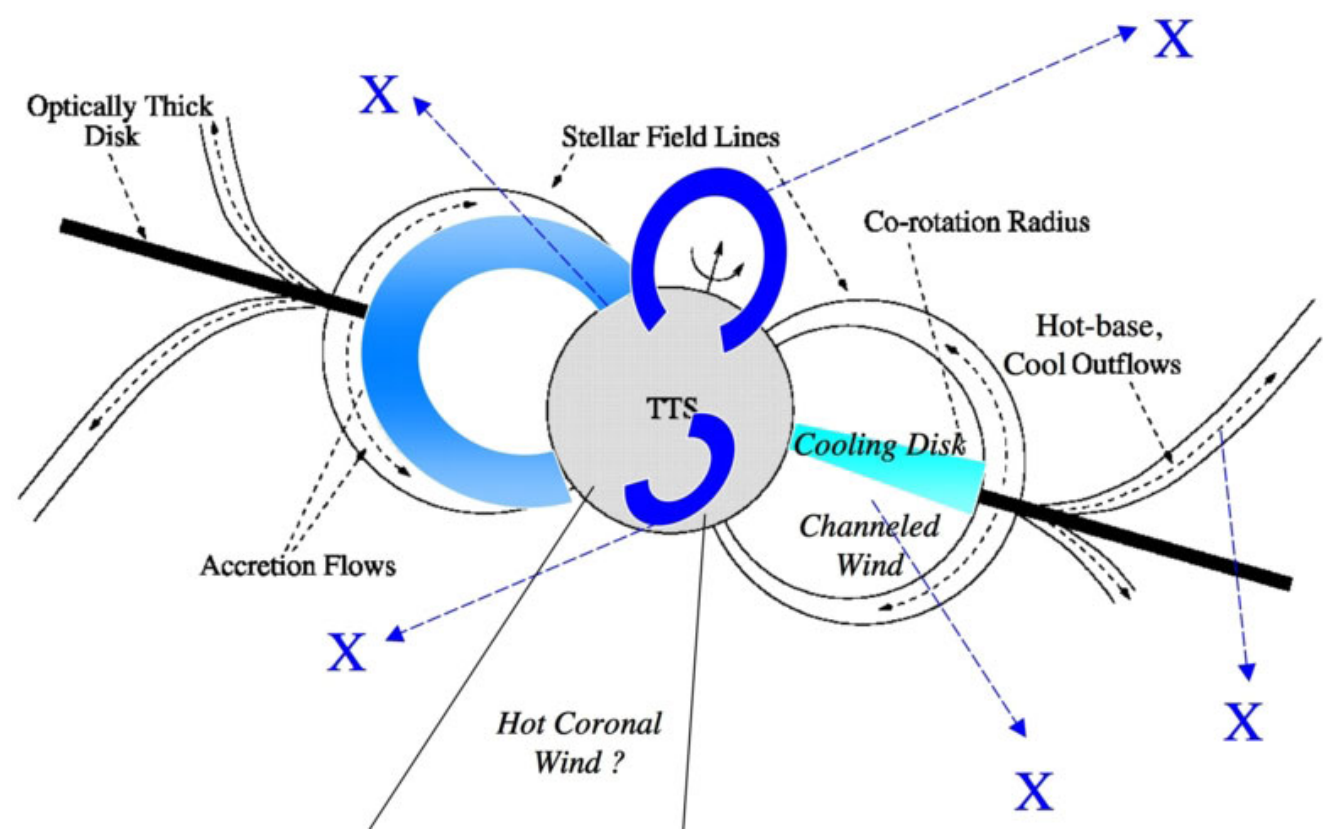

Figure 1. The various X-ray emission regions that may exist in the environment of low- and intermediate-mass stars: magnetic reconnections (on the star or between the star and the disk), and shocks (magnetically channeled winds, accretion on the stellar surface, outflow collisions with the envelope and/or with the interstellar medium, etc.). (Adapted from Stassun 2001)

et al. 2005). Another important observation is the "XMM-Newton extended survey of Taurus" (XEST; PI M. Güdel), a medium exposure ( $30 \mathrm{ksec} /$ field $)$, medium angular resolution (a few arcsec), but large spatial extension (25 XMM fields, i.e., a total of $\sim 5$ square degrees) of the Taurus clouds, yielding over 2400 identifications (mostly 2MASS), of which only $\sim 160$ are characterized to date as young stars (Güdel et al. 2006).

For our purpose here, I briefly comment on three main global results from these satellite observations, restricting the discussion to TTS (for a similar discussion about protostars, see Montmerle 2007). There has been a long-standing debate in the literature about whether there was a statistically meaningful difference in X-rays (luminosities, spectra, etc.) between "classical" and "weak" TTS. The question arose originally simply because the additional extinction of the disk of CTTS (if seen more or less edge-on) would a priori make their spectra harder and their luminosities smaller, and this effect was not seen. On the other hand, we now know that there are in fact three classes of TTS: (i) accreting TTS, which have both evidence for the presence of a disk (mostly from near-IR excess), and for accretion (enhanced $\mathrm{H} \alpha$, CaII, etc., emission: the usual CTTS criterion; see also $\S 5)$; (ii) non-accreting, more evolved TTS, which still have a disk but no evidence for accretion (hereafter "DTTS", for "disk" TTS; unofficial designation !); (iii) even more evolved diskless TTS, which are equivalent to "weak" TTS (evidence neither for a disk nor for accretion). As a result, comparing CTTS and WTTS implies in reality making a distinction between DTTS and "pure" WTTS, and is not the same as comparing the IR-classified "Class II" sources (= CTTS + DTTS) and "Class III" sources (= WTTS).

With the increased sensitivity (i.e., larger sample, lower mass limits, etc.) of the COUP observations, and using only CTTS defined by accretion-related criteria ( $\mathrm{H} \alpha$, CaII), 
Preibisch et al. (2005) found a statistically significant difference of a factor $\sim 2$ in $\mathrm{X}$ ray luminosity between CTTS and WTTS, while for instance Ozawa et al. (2005) found no difference between Class II and Class III young low-mass stars in the $\rho$ Oph cloud core. While, as originally expected, the CTTS turned out to be statistically less Xray luminous than WTTS, this property was found to be uncorrelated with the disk orientation, pointing to a difference in properties linked with the accretion phenomenon itself, and not to the extinction by disk material. We shall discuss this situation in more detail in $\S 5$.

\section{The case of massive (OBA) stars}

As summarized above $(\S 2)$, massive stars (here understood as stars of spectral types earlier than $\mathrm{F}$ ), have radiatively-driven winds, which become stronger and stronger as the effective temperature $T_{\text {eff }}$ increases. A-type stars $\left(T_{\text {eff }} \sim 7,500-10,000 \mathrm{~K}\right)$ are fully radiative. Yet a significant fraction of the A and late B stars $(\sim 5 \%$, see Wade 2005$)$, the so-called Ap and Bp stars, characterized by huge overabundances of heavy elements, are magnetic, with magnetic field strengths $B_{\star}$ reaching several kG (the record being held by Babcock's star, HD 215441, with $B_{\star} \sim 11.5 \mathrm{kG}$ ). The interpretation of overabundances is in terms of element diffusion driven outwards by radiation pressure, but accumulating in the upper photosphere because they are trapped by the magnetic field (e.g., Michaud 2004). Also, gyrosynchrotron radio emission has been detected from a number of $\mathrm{O}$ and B stars (André et al. 1988; Trigilio et al. 2004). The widely accepted interpretation is that of fossil fields brought from the ISM during the early formation and evolutionary stages, although recent work suggest the possibility of an internal, non-convective origin (see MacDonald \& Mullan 2004, and references therein).

On the other hand, X-ray observations have shown that $\mathrm{O}$ and $\mathrm{B}$ stars obey a simple correlation: $L_{X} / L_{b o l} \sim 10^{-7}$, over a wide range of luminosities (Berghöfer et al. 1997). This correlation has been nicely explained by Owocki \& Cohen (1999), in terms of a rather subtle balance between the X-ray emissivity of shocks in the radiatively unstable winds, and extinction as a function of depth in the wind. Yet the COUP observations of a sample of 9 O7 to B3 stars in the vicinity of the Trapezium (the "strong wind" sample of Stelzer et al. 2005) have shown a significant excess of X-ray emission over the nominal $L_{X} / L_{b o l} \sim 10^{-7}$ correlation in three stars, as well as three cases of X-ray rotational modulation in the whole sample. The most spectacular case is that of $\theta^{1}$ Ori $\mathrm{C}$, the most massive star $\left(\mathrm{O} 7, M_{\star} \sim 45 M_{\odot}\right)$ of the Trapezium cluster. The COUP observation confirmed (with much better statistics) the earlier ROSAT result of X-ray modulation (Gagné et al. 1997), with an amplitude of a factor $\sim 2$, at the rotation period of the star $\left(P_{\text {rot }}=15.4 d\right)$. To explain both the high X-ray luminosity of $\theta^{1}$ Ori $\mathrm{C}\left(L_{X} / L_{b o l} \sim 10^{-5}\right)$, and its rotational modulation, Babel \& Montmerle (1997) proposed the existence of a dipolar magnetic field, strong enough to confine the radiative wind, and to channel it along both hemispheres into an equatorial shock. At this shock X-rays are generated, and absorbed by an equatorial disk formed by the cool, post-shock material, resulting in a rotational modulation of the X-ray emission.

This predicted magnetic field was subsequently detected by Donati et al. (2002), with an observed value $B_{\star} \sim 1 \mathrm{kG}$, recently confirmed by Wade et al. (2006). Fig. 2 is a sketch of the so-called "magnetically channeled wind shock" (MCWS) model as introduced by Babel \& Montmerle (1997). More elaborate numerical calculations (e.g., Townsend \& Owocki 2005) have now refined this model, and recent high-resolution X-ray spectra of $\theta^{1}$ Ori C have fully confirmed its validity (Schulz et al. 2003). 


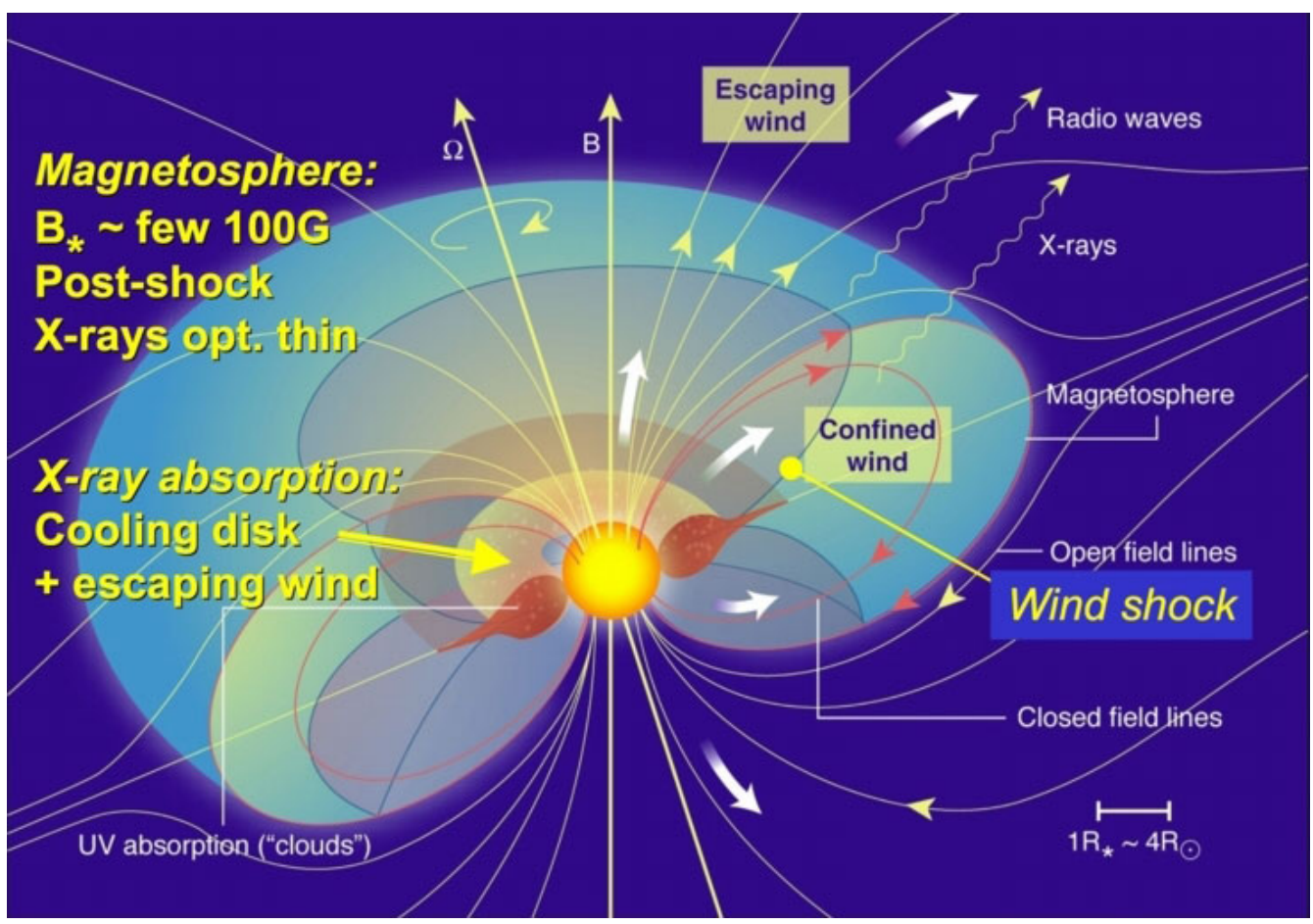

Figure 2. The "magnetically channeled wind shock" (MCWS) model, initially proposed by Babel \& Montmerle (1997) to explain the X-ray rotational modulation of $\theta^{1}$ Ori C. In this model, the radiatively-driven wind of a hot star $(\mathrm{O}, \mathrm{B}$, or A star) is confined by a strong magnetic field, and "self-collides" along the equator, heating the post-shock gas to X-ray energies. Then the shocked wind cools in a dense disk. This disk, in turn, absorbs the X-rays, and if the viewing geometry is favorable, causes a rotational modulation of the X-ray flux. (Adapted from Montmerle 2001)

\section{Magnetospheric accretion and star-disk interactions}

There is widespread support, both observational and theoretical, for "magnetic accretion" in CTTS, i.e., infall of disk material onto the central star, channeled along a dipolar magnetosphere assumed to connecting the star and the inner disk in the vicinity of the corotation radius. However, a number of recent optical observations suggest the existence of a more structured magnetosphere, with discrete "accretion funnels" linking the disk to the star. This departure from cylindrical symmetry may have various natural causes. For instance, "oblique rotator" 3D MHD stationary models (Romanova et al. 2004; also this volume) predict the existence of two main symmetrical accretion funnels and a distortion (warp) of the inner disk structure. The existence of such an inner disk warp in AA Tau, which is seen nearly edge-on, was inferred from multicolor photometry by Bouvier et al. (2007), modulating the X-ray luminosity (Grosso et al. 2007).

What about X-rays in general ? In the preceding sections, we have argued in favor of the widespread interpretation of X-rays from hundreds of young stars in terms of magnetic activity originating in a convective dynamo. However, in a so far handful of cases, the X-rays must be interpreted in terms of emission by shocks from magnetically channeled accretion -reminiscent, in a way, of the MCWS model proposed for some $\mathrm{O}$ stars, but with matter being channeled inwards instead of outwards.

The first case of "non-magnetic activity" X-ray emission from CTTS was reported by Kastner et al. (2002) for TW Hya. Thanks to its proximity ( $d \simeq 60 \mathrm{pc})$, TW Hya is one 
of the brightest CTTS, and high-resolution spectra could be obtained using the Chandra gratings. The analysis revealed an unusually high plasma density $\left(n_{e} \sim 10^{12} \mathrm{~cm}^{-3}\right)$, i.e., an order of magnitude higher than the largest coronal densities, and a very soft spectrum $\left(T_{X}=2 \times 10^{6} \mathrm{~K}\right)$. Kastner et al. (2002) showed that, combined with the absence of time variability during the observations, the data could be interpreted in terms of an accretion shock near the stellar surface at the free-fall velocity of the gas $\left(v_{f f} \sim 200 \mathrm{~km} \mathrm{~s}^{-1}\right)$. To date, two more CTTS (BP Tau and V4046 Sgr) have been found to show X-ray accretion spectral signatures of high plasma densities, determined independently of the temperature on the basis of the He-like NeIX and OVII triplet line ratios (Robrade \& Schmitt 2006; Günther et al. 2006; also Güdel, this volume).

On the other hand, the presence of several accretion funnels connecting the disk to the star is now invoked to explain the factor $\sim 2-3$ deficiency in the X-ray emission of (accreting) CTTS and WTTS ( $\S 3$ ), in terms of additional "self-shielding" provided by the discrete accretion flows (Preibisch et al. 2005).

Another star-disk situation may hold. In the "accretion-ejection" paradigm, corotation, due to magnetic locking, is assumed. However, if magnetic locking is incomplete, i.e., if there is a differential rotation between the star and the disk, there is a possibility of "selfreconnection" within the star-disk magnetic configuration and resulting X-ray emission. It is in this context that Montmerle et al. (2000) explained the "triple flare" observed by $A S C A$ in the Class I protostar YLW15. However, in spite of repeated observations of this star, and even during the two-week-long exposure of COUP, no other case of periodic $\mathrm{X}$-ray flaring on young stars was found. Star-disk interactions, without explicit evidence for periodic X-ray emission, have also been invoked to explain the emission of protostars in general (Preibisch 2004), and arguments in favor of large magnetic structures linking the star and the inner disk have been presented for some Orion TTS (Favata et al. 2005).

\section{The mysterious Herbig stars}

The so-called Herbig AeBe (HAeBe) stars are the young predecessors of intermediatemass stars $\left(M_{\star} \sim 2-4 M_{\odot}\right)$, the future main-sequence A and B stars. They are entirely radiative and have relatively cool effective temperatures $\left(T_{\text {eff }} \sim 5,000-6,000 \mathrm{~K}\right)$, and therefore are not expected to show any sign of magnetic activity, nor a significant wind. Yet, their detection rate in X-rays is quite high $(\sim 76 \%$, Stelzer et al. 2006$)$. The X-ray luminosities, known for many years to reach levels in excess of the brightest TTS (e.g., Zinnecker \& Preibisch 1994), as well as their soft spectra, preclude, contrary to the A stars, the presence of unresolved low-mass companions as the general explanation of their $\mathrm{X}$-ray emission.

In the presumed absence of magnetic fields, some form of accretion shock can be invoked. However, because the stars are more massive than TTS, their free-fall velocities are larger $\left(v_{f f} \approx 500-600 \mathrm{~km} \mathrm{~s}^{-1}\right)$, implying harder X-rays than observed. The high-resolution (XMM RGS) spectrum of AB Aur, the first among HAeBes, with its density-sensitive OVII triplet, does not show evidence for accretion-shock plasma densities (Telleschi et al. 2007), although a Ne excess is present in the low-resolution spectra of several HAeBe stars (Swartz et al. 2005).

UV observations by FUSE may hold the answer. In recent observations of HD 163296 , Deleuil et al. (2005) found that the line profile of several strongly ionized heavy elements gave evidence for a weak wind $\left(\dot{M} \sim 7 \times 10^{-9} M_{\odot} \mathrm{yr}^{-1}, \mathrm{v}_{\mathrm{w}} \sim 300 \mathrm{~km} \mathrm{~s}^{-1}\right)$, but with a much higher emissivity than a normal, freely expanding wind. These authors suggested that, instead of expanding freely, this wind is confined by a large-scale magnetosphere, with a predicted $B_{\star} \sim 700 \mathrm{G}$, in a fashion very similar with the MCWS model of $\theta^{1}$ Ori 
C. Independently, a Zeeman search for magnetic fields in three other HAeBe stars has resulted in one $5 \sigma$ detection of the same order $\left(B_{\star}=450 \pm 93 \mathrm{G}\right)$ (see Hubrig et al. 2007).

Thus, the MCWS model appears promising also to explain the X-ray emission of some HAeBe stars, and implies the existence of magnetic fields in a significant fraction of them, which may be the predecessors to the Ap-Bp stars. In that sense, in X-rays HAeBe stars offer more similarities with OBA stars than with CTTS stars, despite the fact that, like CTTS, they are surrounded by circumstellar disks.

\section{Conclusions}

- Magnetic activity-related X-ray emission, i.e., magnetic reconnection, is by far the most widespread mechanism in convective, low-mass young stars. In a few cases there is indirect evidence for a star-disk reconnection in lieu of the common reconnections between magnetic loops on the star. Thus, as a rule X-rays can be safely taken a proxy for stellar magnetic fields, provided some signatures are checked: hard spectrum, flarelike light curve, coronal densities. Note that the large-scale $\left(R_{c} \approx 0.05 \mathrm{AU}\right)$ dipolar corotating magnetosphere (possibly oblique to the rotation axis) assumed to mediate accretion and ejection cannot be detected in $X$-rays if it is really in a steady state.

- However, there are a few exceptions (three to date) to the general magnetic activity picture. In these few cases, X-rays come from accretion shocks. The signatures are clearly different from the preceding case: soft spectrum, absence of flares, densities much larger than coronal. High densities are best proven by He-like triplets, resolved by grating spectroscopy on $X M M$ or on Chandra.

- Conversely, in the more massive stars, the dominant X-ray emission mechanism is shocks pervading their radiatively unstable winds. X-rays are then precious to probe the inner structure of the wind (density and temperature as a function of radius). However, a large fraction of the OB stars (up to $\sim 50 \%$ in the ONC, Stelzer et al. 2005) show indications of magnetic fields when they are very young. If the magnetic fields are strong enough, then they can confine the wind inside a closed magnetosphere, and the resulting "magnetically channeled" flows from both hemispheres collide and emit shock X-rays, with an X-ray luminosity exceeding that of the standard wind instability mechanism.

- The so-called "Herbig AeBe stars" are commonly referred to as T Tauri stars scaled up in mass, because of the presence of circumstellar disks and/or envelopes. However, from the point of view of X-ray emission, they seem to be more related to massive stars. In particular, at least in some cases of X-ray luminous HAeBe stars, the MCWS model may explain the X/UV emission. For less X-ray luminous HAeBe stars, the presence of a low-mass companion remains the most likely explanation for the X-ray emission.

All in all, we conclude that X-rays from young stars, which are thermal in the $\sim 0.1-10$ $\mathrm{keV}$ range covered by Chandra and $X M M$, always result from some combination of shocks and magnetic fields. On the one hand, magnetic activity dominates in the vast majority of low-mass stars, while on the other hand wind shocks dominate in a majority of high-mass stars. Although the number of "hybrid" cases (i.e., magnetic fields + shocks) is small, they give important insights into the physics of accretion (CTTS), and into the origin and early evolution of magnetic fields in massive stars (Ap-Bp stars).

\section{References}

André, P., et al. 1988, ApJ 335, 940

Babel, J., \& Montmerle, T. 1997, ApJ (Letters) 485, L29

Berghöfer, T.W., et al. 1997, A\& A 322, 167 
Bouvier, J., et al. 2007, A\&A 463, 1017

Brun, A. S., Zahn, J.-P. 2006, A\&SA 457, 665

Deleuil, M., et al. 2005, A\&ऽA 429, 247

Donati, J.-F., et al. 2002, MNRAS 333, 55

Donati, J.-F., et al. 2007, in 14th Cambridge Workshop on Cool Stars, Stellar Systems, and the Sun, Ed. G. van Belle, ASP Conf. Ser., in press (astro-ph/0702159)

Favata, F., et al. 2005, ApJ(Suppl.) 160, 469

Feigelson, E. D., \& Montmerle, T. 1999, ARA\&A 37, 363

Gagné, M., et al. 1997, ApJ (Letters) 478, L87

Getman, K. V., et al. 2005, ApJS 160, 319

Grosso, N., et al. 2007, A\&A in press

Güdel, M. 2004, Astr.Ap.Rev. 12, 71

Güdel, M., et al. 2006, A\&BA in press (astro-ph/0609160)

Günther, H. M., et al. 2006, A\&SA 459, L29

Hillenbrand, L. A. 2006, in A Decade of Discovery: Planets Around Other Stars, Ed. M. Livio, STScI Symposium Ser., 19, in press

Hubrig, S., et al. 2007, A\&A 463, 1039

Kastner, J. H., et al. 2002, ApJ 567, 434

Kudritzki, R.-P., \& Puls, J. 2000, ARA\&A 38, 613

MacDonald, J., Mullan, D. J. 2004, MNRAS 348, 702

Micela, G., Favata, F. 2005, Sp. Sci. Rev. 108, 577

Michaud, G. 2004, in The A-Star Puzzle, Eds. J. Zverko et al. IAUS 224 (Cambridge, UK: Cambridge University Press), p.173

Montmerle, T. 2001, Science 293, 2409

Montmerle, T. 2007, Mem. Soc. Astr. It. in press

Montmerle, T., et al. 2000, ApJ 532, 1097

Owocki, S.P., \& Cohen, D.H. 1999, ApJ 520, 833

Ozawa, H., Grosso, N., Montmerle, T. 2005, A\&A 438, 963

Peres, G., Orlando, S., Reale, F. 2004, ApJ 612, 472

Preibisch, T. 2004, A\& $A$ 428, 569

Preibisch, T., et al. 2005, ApJS 160, 401

Pudritz, R. E., et al. 2007, in Protostars and Planets V, Eds. B. Reipurth, D. Jewitt, \& K. Keil (Tucson: University of Arizona Press), p.277

Robrade, J., Schmitt, J. H. M. M. 2006, A\&\&A 449, 737

Romanova, M. M., et al. 2004, ApJ 610, 920

Schulz, N. S., et al. 2003, ApJ 595, 365

Stassun, K. G. 2001, in From Darkness to Light, Eds. T. Montmerle \& P. André, ASP Conf. Ser., 243, p.599

Stelzer, B., Flaccomio, E., Montmerle, T., et al. 2005, ApJS 160, 557

Stelzer, B., et al. 2006, A\&GA 457, 223

Strassmeier, K. G., Rice, J. B. 2006, A\&A 460, 751

Swartz, D. A., et al. 2005, ApJ 628, 811

Telleschi, A., et al. 2007, A\&GA in press (astro-ph/0610456)

Townsend, R. H. D., Owocki, S. P. 2005, MNRAS 357, 251

Trigilio, C., et al. 2004, A\&A 418, 593

Wade, G.A. 2005, in Element Statification in Stars, Eds. G. Alecian, O. Richard, \& S. Vauclair, EAS Publ. Series, 17, p.227

Wade, G. A., et al. 2006, A\&A 451, 195

Wolk, S. J.; et al. 2005, ApJ 160, 423

Yang, Hao, Johns-Krull, C. M., Valenti, J. A. 2007, ApJ 133, 73

Zinnecker, H., Preibisch, T. 1994, A\&A 292, 152 